\title{
Manuka Honey Modulates the Inflammatory Behavior of a dHL-60 Neutrophil Model under the Cytotoxic Limit
}

\author{
Benjamin A. Minden-Birkenmaier $\mathbb{D}^{1},{ }^{1}$ Kasyap Cherukuri, ${ }^{1}$ Richard A. Smith, \\ Marko Z. Radic $(1),{ }^{3}$ and Gary L. Bowlin $\left(\mathbb{1}^{1}\right.$ \\ ${ }^{1}$ Department of Biomedical Engineering, University of Memphis, 3806 Norriswood Ave., Memphis, TN 38152, USA \\ ${ }^{2}$ Department of Orthopaedic Surgery \& Biomedical Engineering, University of Tennessee Health Science Center, \\ 956 Court Ave., Memphis, TN 38163, USA \\ ${ }^{3}$ Department of Microbiology, Immunology and Biochemistry, University of Tennessee Health Science Center, \\ 858 Madison Ave., Memphis, TN 38163, USA
}

Correspondence should be addressed to Gary L. Bowlin; glbowlin@memphis.edu

Received 26 November 2018; Accepted 28 January 2019; Published 25 February 2019

Academic Editor: Nicholas Dunne

Copyright (C) 2019 Benjamin A. Minden-Birkenmaier et al. This is an open access article distributed under the Creative Commons Attribution License, which permits unrestricted use, distribution, and reproduction in any medium, provided the original work is properly cited.

\begin{abstract}
Recent work has shown that Manuka honey, an increasingly popular wound additive with potent antibacterial properties, also has anti-inflammatory properties. However, little research has been done examining its effect on neutrophils. This study investigates the hypothesis that Manuka honey reduces neutrophil superoxide release and chemotaxis and reduces the activation of the inflammatory nuclear factor- $\kappa \mathrm{B}(\mathrm{NF}-\kappa \mathrm{B})$ signaling pathway under honey's cytotoxic limit. A differentiated HL-60 cell line was used as a neutrophil model and cultured in various concentrations of Manuka honey for 3 and 24 hours to measure cytotoxicity via mitochondrial activity and visual trypan-exclusion count. Cytochrome $\mathrm{C}$ and Boyden chamber assays were used to measure the effect of Manuka honey on superoxide release and chemotaxis toward fMLP, respectively. Additionally, a Western blot for NF$\kappa \mathrm{B}$ inhibitor $\alpha(\mathrm{I} \kappa \mathrm{B} \alpha)$ was performed to measure Manuka honey's effect on the NF- $\kappa \mathrm{B}$ pathway via I $\kappa \mathrm{B} \alpha$ phosphorylation. The results indicate a cytotoxic limit of $3-5 \% \mathrm{v} / \mathrm{v}$. The presence of $1 \%$ honey decreased superoxide release at 24 hours. The $0.5,1$, and $3 \%$ honey concentrations reduced chemotaxis and $\mathrm{I} \kappa \mathrm{B} \alpha$ phosphorylation in a dose-dependent fashion. These results suggest that Manuka honey significantly reduces neutrophil recruitment and inflammatory behavior in the wound site in a dose-dependent fashion under the cytotoxic limit.
\end{abstract}

\section{Introduction}

Studies have demonstrated that topical application of honey to wounds promotes wound closure, induces an osmotic gradient which cleanses the wound via fluid movement, reduces wound inflammation, and inhibits the growth of a range of bacteria varieties [1-4]. The high concentration of sugars in the honey creates an osmotic gradient that pulls fluid from the subcutaneous tissue up through the wound area, flushing necrotic debris from the wound site and carrying nutrients and oxygen from the surrounding area into the damaged tissue [5]. Additionally, this gradient helps to remove excess fluid from the wound environment, which has been shown to impede bacterial growth [6]. Flavonoids within the honey scavenge free oxygen radicals, reducing inflammation and minimizing tissue damage [7-9]. Previous work by Alvarez-Suarez et al. has analyzed the phenolic content of Manuka honey via HPLC-MS, and it is theorized that these components improve the intracellular antioxidant response [10]. In addition, honey's hydrogen peroxide content acts as an antiseptic against many types of bacteria [11-13]. These properties and others have been reviewed in detail in previously published literature $[5,14-20]$.

The anti-inflammation and prohealing properties of Manuka honey have led some groups to incorporate it as an additive within biomaterials such as tissue engineering templates [21-25]. As the implantation of these templates requires the creation of a wound site and the associated increase in 
neutrophil presence, the effect of Manuka honey on neutrophil activity is relevant to this line of research. Excessive neutrophil inflammatory activity has been implicated in the initiation of fibrosis, which can impede tissue-biomaterial integration [26]. The ability of Manuka honey to modulate such neutrophil inflammatory activity would increase its usefulness as a template additive. Of particular importance to this research are potential cytotoxic effects of the honey, which could inhibit cell infiltration and proliferation within these templates. As such, it is necessary to determine the concentration at which honey becomes cytotoxic to neutrophils and to investigate a range of honey concentrations to determine the optimum loading and release levels for tissue engineering templates.

In this study, a specific variety of honey termed Manuka honey is used. In addition to the effects described above, Manuka honey contains a methylglyoxal component which imbues it with additional antimicrobial activity [4, 27]. This methylglyoxal component is primarily responsible for the Unique Manuka Factor (UMF), a term used by the industry to describe the heightened antimicrobial activity of Manuka honey. After Manuka honey is collected, it is subject to a bacterial inhibition test, and the UMF is defined as the concentration of phenol necessary to achieve the bacterial inhibition of that Manuka honey sample (for instance, Manuka honey with a UMF of 15 would exhibit the same bacterial inhibition as $15 \%$ phenol) [28]. This test is standardized and used across the industry to compare the antimicrobial effects of various Manuka honey products $[23,29,30]$. As the properties of Manuka honey can vary slightly based on area of collection and processing parameters, this test allows for a standardized measurement of the honey's bacterial inhibition properties.

Although the role of neutrophils has been classically considered to be primarily phagocytic, recent research has demonstrated the ability of these cells to regulate wound healing through the release of growth factors, chemo/cytokines, and proteases [31]. These cells arrive through the bloodstream, travel via chemotaxis to the wound site soon after the occurrence of injury, and begin fighting bacterial invasion via phagocytosis, superoxide release, and the extrusion of neutrophil extracellular traps (NETosis) [32]. Additionally, they release a wide variety of factors that recruit more neutrophils, macrophages, and other inflammatory cells and also amplify the overall inflammatory response [33]. While this proinflammatory neutrophil activity is effective at fighting bacterial invasion, the factors released by these cells can damage native tissue, impairing wound healing [34]. However, in addition to this proinflammatory behavior, neutrophils also have the ability to exhibit anti-inflammatory, proresolution behaviors $[35,36]$. These behaviors include inhibiting additional neutrophil recruitment to the wound site, downregulating the degranulation of mast cells, releasing anti-inflammatory IL10 , promoting angiogenesis, and inhibiting T-cell activation [34, 35, 37, 38]. The balance of these neutrophil behaviors is important in both promoting acute inflammation and transitioning the wound from inflammation to resolution and healing, and thus avoiding chronic inflammation [32, 35]. Given the importance of neutrophils in controlling wound inflammation, as well as the growing clinical use of Manuka honey as a wound additive with demonstrated anti-inflammatory properties, the effect of Manuka honey on neutrophil behavior is of scientific interest [39].

One important indicator of the activation of the inflammation response of the neutrophil is the phosphorylation of $\mathrm{I} \kappa \mathrm{B} \alpha$, a regulatory protein which inhibits the inflammatory nuclear transcription factor $\mathrm{NF}-\kappa \mathrm{B}$ by trapping it in the cytoplasm. NF- $\kappa \mathrm{B}$ is a transducer for many inflammatory pathways [40-43]. These signal cascades trigger the phosphorylation of $\mathrm{I} \kappa \mathrm{B} \alpha$, which is bound to NF- $\kappa \mathrm{B}$. As $\mathrm{I} \kappa \mathrm{B} \alpha$ is phosphorylated, it releases the NF- $\kappa \mathrm{B}$ molecules, allowing them to enter the nucleus and bind to promoter regions in the DNA. By binding to these regions, NF- $\kappa$ B upregulates the transcription of inflammation response genes [44-46].

In this study, the human leukemia HL-60 cell line was differentiated and used as a model of the neutrophil. This neutrophil model has been extensively characterized [4749]. Certain behaviors of primary neutrophils can vary substantially from donor to donor, including substrate adherence, chemotaxis, cytokine release, and damage to surrounding cells/tissues [50-52]. Utilizing the dHL-60 model eliminates this variability and provides a reliable standard that can be used by others in the field. These differentiated cells were polarized with lipopolysaccharide (LPS) and formylmethionine-leucyl-phenylalanine (fMLP) as inflammatory stimuli or transforming growth factor $\beta 1$ (TGF- $\beta$ ) as an anti-inflammatory stimulus. These cells were cultured in a range of concentrations of Manuka honey, and their behavior was characterized with regard to cytotoxicity, superoxide release, chemotaxis, and $\mathrm{I} \kappa \mathrm{B} \alpha$ phosphorylation.

The hypothesis of this study is that Manuka honey reduces neutrophil inflammatory behavior, specifically superoxide release, chemotaxis to the bacterial signal fMLP, and $\mathrm{I} \kappa \mathrm{B} \alpha$ phosphorylation, when present under the cytotoxic limit.

\section{Materials and Methods}

2.1. HL-60 Culture and Differentiation. HL-60s were purchased from the American Type Culture Collection (ATCC, CCL 240, Manassas, VA, USA) and cultured at a cell density range of $2 \times 10^{5}$ to $1 \times 10^{6}$ cells per $\mathrm{mL}$ in culture media consisting of RPMI (Hyclone, Logan, UT, USA) with 10\% v/v non-heat-inactivated fetal bovine serum (FBS) (Hyclone), $1 \%$ v/v penicillin/streptomycin (Pen/Strep) (Corning, NY, USA), and 1\% L-glutamine (Gibco, Billings, MT, USA) (referred to as culture medium). Cells were incubated at $37^{\circ} \mathrm{C}$ in a $5 \%$ $\mathrm{CO}_{2}$ environment in T-25 and T-75 culture flasks (Thermo Scientific, Rochester, NY, USA). Medium was changed every 3-4 days and cells were passaged when cell density reached $5 \times 10^{5}$ cells $/ \mathrm{mL}$. Cells were used for passage numbers up to 30. These cells were differentiated to a neutrophil-like phenotype by adding $1.25 \%$ dimethylsulfoxide (DMSO) (Fisher Scientific, Hampton, NH, USA) to the culture medium for six days, replenishing the medium/DMSO on the third day. This procedure has been validated in previous studies [27, 28].

2.2. Confirmation of Differentiation. Differentiation was confirmed morphologically by permeabilizing with $0.17 \mathrm{mM}$ Triton X-100 (Fisher Scientific) for 5 minutes, then fixing in 
$4 \%$ buffered paraformaldehyde (Fisher Scientific) and staining with 4,6-diamidino-2-phenylindole (DAPI) (NucBlue Fixed Cell Stain ReadyProbes reagent) for 5 minutes at stock concentration and phalloidin-conjugated Alexa Fluor 488 (ActinGreen 488 ReadyProbes reagent) (both from Invitrogen, Carlsbad, CA, USA) for 30 minutes also at stock concentration according to the manufacturer's protocols. Cells were imaged with an Olympus microscope (model BX34F) with an attached Olympus DP73 digital color camera and Olympus U-HGLGPS fluorescent light source (Olympus, Shinjuku, Tokyo, Japan). The percentage of differentiated cells (kidneyshaped nucleus) was calculated to be $69 \%$, comparable to the percentage reported in literature (see Supplemental Section 1) $[47,53]$.

2.3. Cell Stimulation. Differentiated HL-60s (dHL-60s) were incubated in the presence of inflammatory and antiinflammatory stimuli. Proinflammatory responses were elicited by incubating dHL-60s with $1 \mu \mathrm{g} / \mathrm{mL}$ LPS (Invivogen, San Diego, CA, USA) for 90 minutes, then adding $10^{-7} \mathrm{M}$ fMLP (Sigma Aldrich, St. Louis, MO, USA) immediately prior to use. Work by Nath et al. has previously shown these concentrations of LPS and fMLP with a 90-minute polarization step to effectively stimulate superoxide release in neutrophils [54]. Anti-inflammatory responses were elicited by incubating dHL-60s with $2 \mathrm{ng} / \mathrm{mL}$ TGF- $\beta 1$ (Gibco) for 24 hours prior to use. While TGF- $\beta$ levels on the order of $\mathrm{pg} / \mathrm{mL}$ have been shown to elicit neutrophil migration, levels on the order of $2 \mathrm{ng} / \mathrm{mL}$ have been shown to exist within the healing wound environment during peak neutrophil recruitment $[55,56]$. Unstimulated dHL-60s were used as an additional experimental control.

2.4. Mitochondrial Activity Assay. To begin this assay, 400,000 of non-stimulated (NS), inflammatory stimulated (LPS + fMLP), and anti-inflammatory stimulated (TGF- $\beta$ ) dHL-60s were seeded in $150 \mu \mathrm{L}$ culture media in a 96well plate in $0,0.1,0.5,1,3,5,10$, and $20 \% \mathrm{v} / \mathrm{v}$ concentrations of Manuka honey/culture medium (UMF 12+, Manuka Guard, Monterey, CA, USA, density of $1.51 \mathrm{~g} / \mathrm{mL}$ ) alongside honey/medium blanks containing no cells. At 0,3 , and 24 hours, $30 \mu \mathrm{L}$ of MTS solution (CellTiter 96 Aqueous One Solution Cell Proliferation Assay, Promega, Madison, WI, USA) was added to each well including the honey/medium blanks. After incubation at $37^{\circ} \mathrm{C}$ for 1 hour, the absorbances of the samples were read at $490 \mathrm{~nm}$ using a SpectraMax i3x Multi-Mode Detection plate reader (Molecular Devices, Sunnyvale, CA, USA). The absorbances of the honey/medium blanks were subtracted from the corresponding samples, and the results were expressed as relative mitochondrial activity. Statistical significance was measured via a two-way analysis of variance (ANOVA) with a Holm-Sidak post hoc $(\alpha=0.05)$.

2.5. Trypan-Exclusion Assay. To conduct this assay, 400,000 of NS, LPS + fMLP-stimulated, and TGF- $\beta$-stimulated dHL60s were seeded in $150 \mu \mathrm{L}$ in a 96-well plate in $0,0.1$, $0.5,1,3,5,10$, and $20 \% \mathrm{v} / \mathrm{v}$ of Manuka honey/medium for 3 and 24 hours. After culturing for their respective time periods, the liquid and cells from each well were pipetted into microcentrifuge tubes. Then, $30 \mu \mathrm{L}$ of trypsin (Gibco) was pipetted into each well and incubated for 5 minutes at $37^{\circ} \mathrm{C}$ to remove any remaining adherent cells. After the incubation period, the trypsin was pipetted out of each well and added to the corresponding microcentrifuge tube, which was centrifuged at $200 \mathrm{X} \mathrm{G}$ for 10 minutes. Supernatants were discarded, and cells were resuspended in $75 \mu \mathrm{L}$ culture media with $75 \mu \mathrm{L}$ trypan blue (Gibco). The numbers of viable and nonviable cells in each sample were counted with a Countess II FL Automated Cell Counter (Invitrogen). A twoway ANOVA with a Holm-Sidak post hoc was performed to test for statistical significance between groups $(\alpha=0.05)$.

2.6. Superoxide Production Assay. For this study, 150,000 NS, LPS + fMLP-stimulated, and TGF- $\beta$-stimulated dHL60s were seeded in $150 \mu \mathrm{L}$ culture media in 96-well plates in $0,0.1,0.5,1,3,5,10$, and $20 \% \mathrm{v} / \mathrm{v}$ of honey with $100 \mu \mathrm{M}$ ferricytochrome C (Sigma Aldrich) in accordance with a previously defined procedure $[54,57,58]$. After 1,3 , and 24 hours of culture, the absorbance at $550 \mathrm{~nm}$ was measured using the Spectramax plate reader. Honey/medium blanks of each honey concentration were run alongside the cell samples, and the absorbances of these blanks were subtracted from the cell samples to get the absorbance due to ferricytochrome $\mathrm{C}$ reduction. The results were displayed as relative superoxide production. Statistical significance was measured via a twoway ANOVA with a Holm-Sidak post hoc $(\alpha=0.05)$.

2.7. Chemotaxis Assay. Polystyrene transwell inserts with a 6.5 - $\mathrm{mm}$ diameter polyester membrane perforated by $3.0 \mu \mathrm{m}$ diameter pores (Costar, Kennebunk, ME, USA) were used to measure chemotaxis via an adaptation of a previously defined method [59]. Briefly, 500,000 NS dHL-60s were seeded in the top inserts in $100 \mu \mathrm{L}$ of culture medium, and $650 \mu \mathrm{L}$ of culture medium with $50 \mathrm{nM}$ fMLP and $0,0.5,3$, and $20 \%$ honey was placed in the bottom chamber (fluid levels were the same height when the top inserts were placed onto the bottom chambers to avoid net fluid flow from one chamber to another). One additional control was run of NS cells seeded in top inserts above chambers containing medium with no honey or fMLP to establish the amount of cell movement that happens in the absence of a chemokine. Samples were incubated for 3 hours. At the end of the incubation period, the top inserts were removed from the bottom chambers. The contents of each top and bottom chamber were pipetted out into microcentrifuge tubes. $30 \mu \mathrm{L}$ of trypsin was added to each top chamber, and $650 \mu \mathrm{L}$ of trypsin was added to each bottom chamber, and then the plates were incubated at $37^{\circ} \mathrm{C}$ for 5 minutes. Trypsin was then removed from each top and bottom chamber and added to the respective microcentrifuge tubes, which were then centrifuged at $200 \mathrm{x}$ G for 10 minutes. Supernatants were removed and discarded, and each sample was resuspended in $75 \mu \mathrm{L}$ of RPMI and $75 \mu \mathrm{L}$ of trypan blue. The numbers of viable and nonviable cells in each sample were then counted with a Countess II FL Automated Cell Counter (Invitrogen). Statistical significance was measured via a two-way ANOVA with a Holm-Sidak post hoc $(\alpha=0.05)$. 
2.8. IKB $\alpha$ Phosphorylation Western Blot. Preliminary experimentation indicated that peak IKB $\alpha$ phosphorylation occurs 38 minutes after the addition of LPS and fMLP at the same time to the culture medium (results shown in Supplemental section 2). Accordingly, in this set of experiments the LPS was added with the fMLP at time 0 , rather than 90 minutes before time 0 as was done in the above experiments. TGF$\beta$ was still added to its group 24 hours before time 0 . NS, LPS + fMLP, and TGF- $\beta$-stimulated dHL-60s were seeded at 400,000 cells per well in $150 \mu \mathrm{L}$ of culture media in 96-well plates with $0,0.5$, and $3 \% \mathrm{v} / \mathrm{v}$ Manuka honey and incubated for 38 minutes. Plates were then placed on ice and cells were removed and lysed in $50 \mu \mathrm{L}$ radioimmunoprecipitation buffer (RIPA buffer, Thermo Scientific), using trypsin to remove remaining cells. Lysates were centrifuged at 13,000 x G at $4^{\circ} \mathrm{C}$ for 15 minutes to pellet the cell membrane detritus, and the supernatant was saved. Samples were denatured with lithium dodecyl sulfate (LDS) sample buffer and dithiothreitol (DTT) reducing agent (both from Invitrogen) at $70^{\circ} \mathrm{C}$ for 10 minutes. Proteins were subjected to gel electrophoresis using 3-8\% Tris-Acetate gels (Invitrogen) and transferred to polyvinylidene difluoride membranes (Millipore, Burlington, MA, USA). Membranes were washed 5 times with Trisbuffered saline (TBS, Thermo Scientific), blocked with TBS blocking buffer (Thermo Scientific) for an hour, and then incubated overnight at $4^{\circ} \mathrm{C}$ in mouse anti-human IKB $\alpha$ primary antibody (MAB4299, R\&D Systems, Minneapolis, $\mathrm{MN}, \mathrm{USA}$ ) at a $0.1 \mu \mathrm{g} / \mathrm{mL}$ concentration and rabbit antihuman phospho-IKB $\alpha$ (S32/S36) primary antibody (AF4809, $\mathrm{R} \& \mathrm{D}$ Systems) at a $1 \mu \mathrm{g} / \mathrm{mL}$ concentration in TBS blocking buffer. Following 5 washes in TBS with $0.1 \%$ Tween 20 (Fisher Scientific), the membrane was incubated at room temperature in IRDye 680RD Donkey anti-mouse secondary (P/N 925-68072) and IRDye 800CW Donkey anti-rabbit secondary (P/N 925-32213) (both from Li-Cor Biosciences, Lincoln, NE, USA) at a 1:20,000 dilution in TBS blocking buffer with $0.1 \%$ Tween 20 and $0.01 \%$ sodium dodecyl sulfate (Fisher Scientific) for one hour. The membrane was washed 3 times in TBS with $0.1 \%$ Tween 20 , one time in TBS, and then scanned on an Odyssey CLx infrared imaging system (Li-Cor Biosciences). The relative fluorescence of the $800 \mathrm{~nm}$ and $700 \mathrm{~nm}$ channels was calculated for the relevant bands of each sample, subtracting out background fluorescence from the area around the bands using Image Studio ${ }^{\mathrm{TM}}$ version 5.2 software. Samples were run in groups of 3 concurrent lanes on two separate Western blots (total of 6 samples per group). Each Western blot had 3 lanes of non-stimulated dHL-60s cultured without honey, and the relative fluorescence ratio of all other samples was normalized to this control. Statistical significance was measured via a two-way ANOVA with a Holm-Sidak post hoc $(\alpha=0.05)$.

\section{Results}

3.1. MTS Mitochondrial Assay. Figure 1 shows the mitochondrial activity of cells of all three stimulation groups incubated at various concentrations of honey in (a) the first hour of incubation, (b) from hours 3 to 4, and (c) from hours 24 to 25. as shown by the black asterisks, a statistically significant decrease in mitochondrial activity begins at 3\% honey and becomes more pronounced as honey concentration increases. This effect becomes stronger at the 3-4-hour and 24-25-hour time windows, suggesting a cytotoxic effect of the honey that begins in the range of 3-5\% and becomes more accentuated at higher honey concentrations. These data sets also show that the mitochondrial activity of the TGF- $\beta$-stimulated cells decreases relative to the other two groups at the 3-4-hour and 24-25-hour time windows. This finding could indicate that the anti-inflammatory effect of the TGF- $\beta$ stimulation decreases the overall cellular metabolism, although further studies would be necessary to confirm this hypothesis. As shown in Figure 1(c), the mitochondrial activity of the NS group is significantly upregulated at $0.5 \%$ and $1 \%$ honey, possibly indicating increased cellular metabolism of this phenotype at these intermediate honey concentrations.

3.2. Trypan Exclusion Assay. Figure 2 displays the number of viable (trypan-excluding) and nonviable (non-trypanexcluding) cells of each stimulation group after 3 and 24 hours of culture with various concentrations of honey. At the 3-hour timepoint, there is a significant decrease in viable (a) NS, (c) LPS + fMLP, and (e) TGF- $\beta$-stimulated cells and an increase in nonviable cells of each group at $20 \%$ honey, with this trend beginning at $5 \%$ honey in the TGF- $\beta$ group and $10 \%$ honey in NS group, indicating cytotoxicity at these concentrations. At the 24-hour timepoint, this significant dropoff in viable (d) LPS + fMLP-stimulated cells and increase in nonviable cells are seen at 3\% honey and up, and in (b) NS and (f) TGF- $\beta$-stimulated cells, this effect happens at $5 \%$ honey and up. These data confirm the findings from the mitochondrial activity data that there is weak cytotoxicity in the 3-5\% honey range and strong cytotoxicity at concentrations higher than $5 \%$ honey. The counts of the TGF- $\beta$ group at 3 and 24 hours also confirm that the drop in mitochondrial activity that occurred in this group at 3-4 hours and 24-25 hours is not a result of increased cell death of this phenotype, but rather decreased mitochondrial activity of viable cells relative to the other two phenotypes.

3.3. Superoxide Production. Figure 3 shows the superoxide production of all three stimuli groups after 1, 3, and 24 hours of culture. As expected, the LPS + fMLP samples have significantly greater superoxide production at $0 \%$ honey at 1 and 3 hours relative to the other two stimuli groups (positive control). However, this difference decreases as honey concentration increases and superoxide production increases in the NS and TGF- $\beta$ groups. This trend indicates that, within the first three hours, concentrations of $3 \%$ honey and above stimulate superoxide production in equal to or in excess of the superoxide production stimulated by LPS and fMLP in both NS and TGF- $\beta$-stimulated neutrophils and also further stimulate superoxide production in the LPS + fMLP group. However, as shown in Figure 3(c) at the 24-hour timepoint, there is an opposite trend with regard to honey concentration. At levels of 3\% honey and above, superoxide levels significantly decrease in all three groups relative to the $0 \%$ honey control. Part of this decrease is likely due 


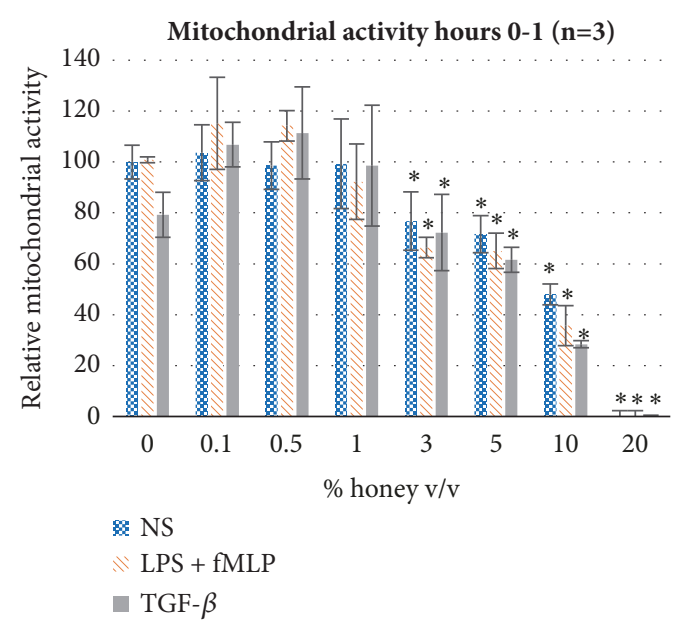

(a)

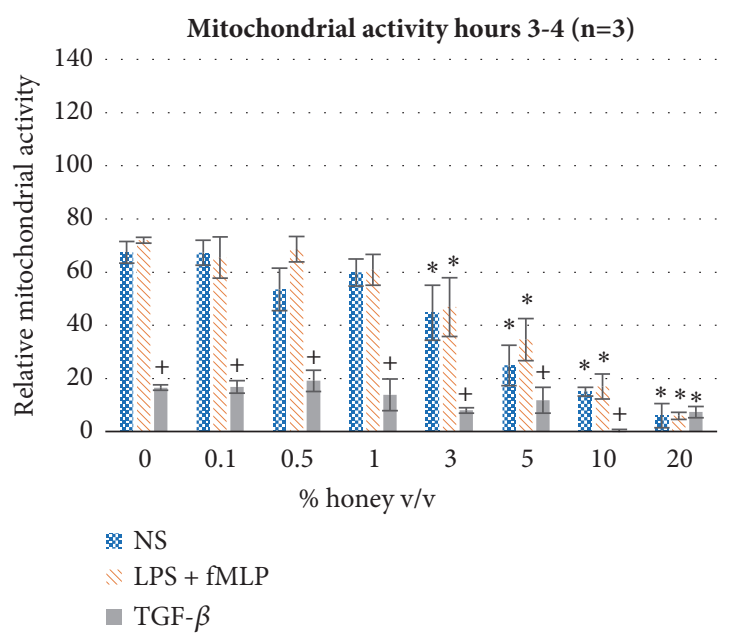

(b)

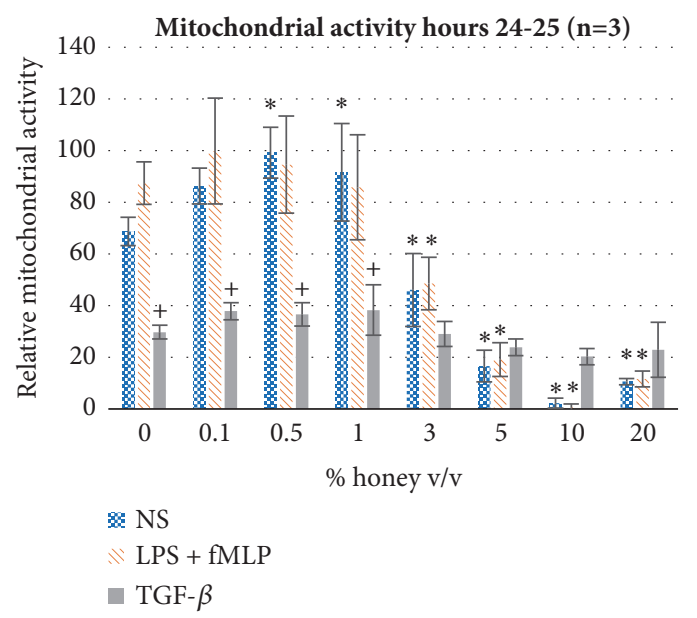

(c)

FIGURE 1: Mitochondrial activity of NS, LPS/fMLP, and TGF- $\beta$-stimulated dHL-60s at various concentrations of honey for hours (a) $0-1$, (b) 3-4, and (c) 24-25 of culture. Values are normalized relative to the 0-1-hour NS 0\% honey mitochondrial activity. * indicates statistical significance from the respective $0 \%$ honey value of that phenotype/timepoint, and + indicates statistical significance from the other two phenotypes at that honey concentration and timepoint. $\alpha=0.05$, measured via two-way ANOVA with Holm-Sidak post hoc.

to the cytotoxic effect of these higher honey concentrations (addressed in the discussion section). Additionally, the TGF$\beta$ group has lower superoxide production than the other groups at intermediate honey concentrations $(0.5 \%$ and $1 \%)$ at 1 and 3 hours (significant at $0.5 \%$ honey at the 3 -hour timepoint) and has significantly less superoxide production relative to the other two phenotypes at the 24-hour timepoint at honey concentrations of $1 \%$ and lower. However, these cells did significantly increase their superoxide production at $3 \%$, $5 \%$, and $10 \%$ honey at the 1 -hour mark and at $5 \%$ honey at the 3 -hour timepoint, relative to their $0 \%$ honey control. At the 24-hour timepoint, the total cell numbers (trypan-excluding and not trypan-excluding) at 5\% honey are lower than the seeded number. This discrepancy could be due to the destruction of nonviable cells or the phagocytosis of the nonviable cells by the live ones. However, additional experimentation would be required to confirm this assumption.
3.4. Chemotaxis. Figure 4 shows the results of the transwell chemotaxis assay. These results show the total amount of cells, both viable and nonviable, present in the top and bottom wells after the 3-hour incubation period. The significant decrease in migration in the no fMLP control relative to the $0 \%$ honey sample indicates that the cells are migrating from the top wells to the bottoms in response to the presence of fMLP throughout the 3-hour experiment. Similarly, the significant decrease in migration in the $0.5 \%, 3 \%$, and $20 \%$ honey samples indicates that Manuka honey reduces this chemotactic response, decreasing the amount of migration to a level at or below the random walk level seen in the no fMLP control. The $20 \%$ honey sample had a significant decrease in the number of cells in both the top and bottom chambers, most likely due to the cytotoxicity of this honey concentration and the effect described above with respect to Figure 2. 


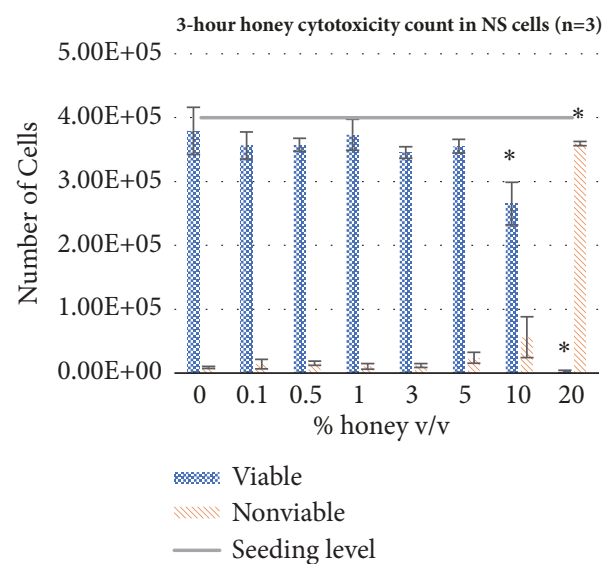

(a)

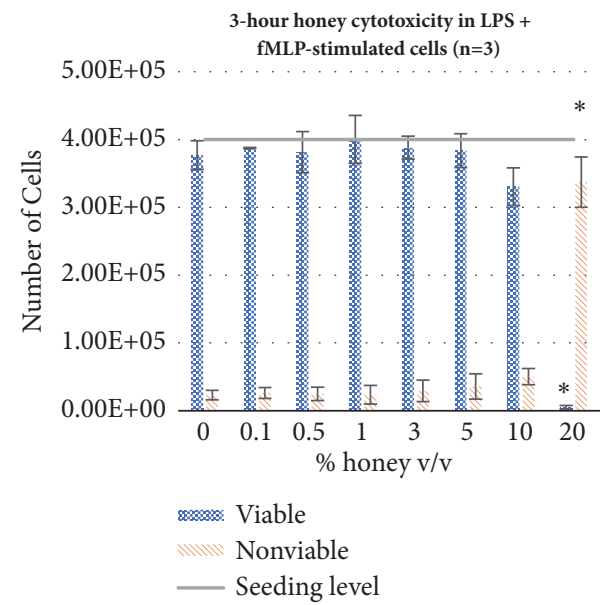

(c)

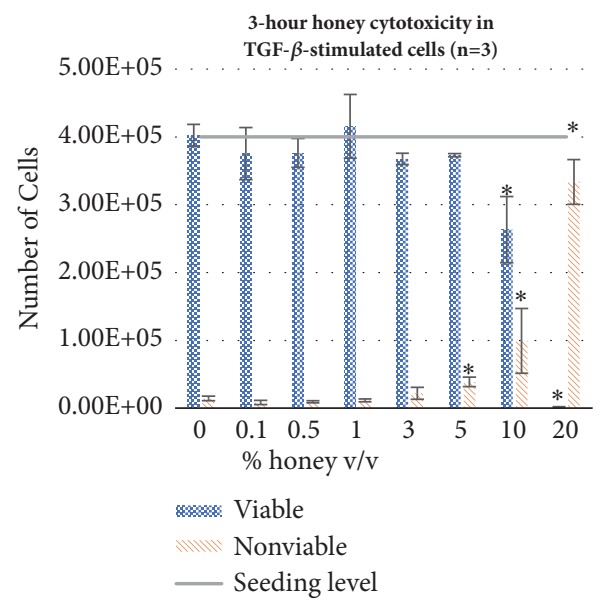

(e)

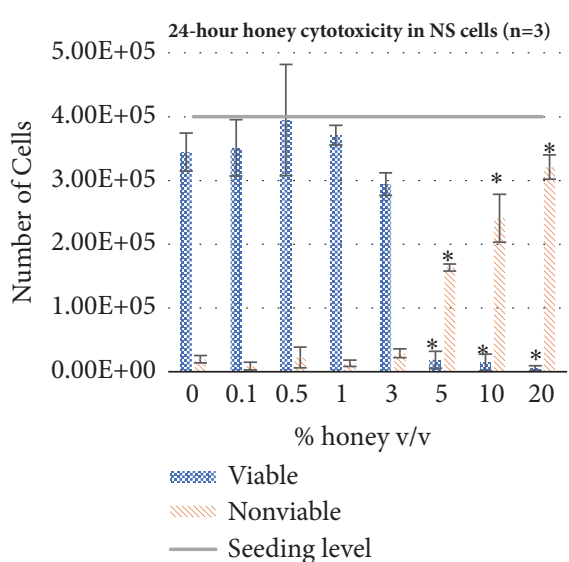

(b)

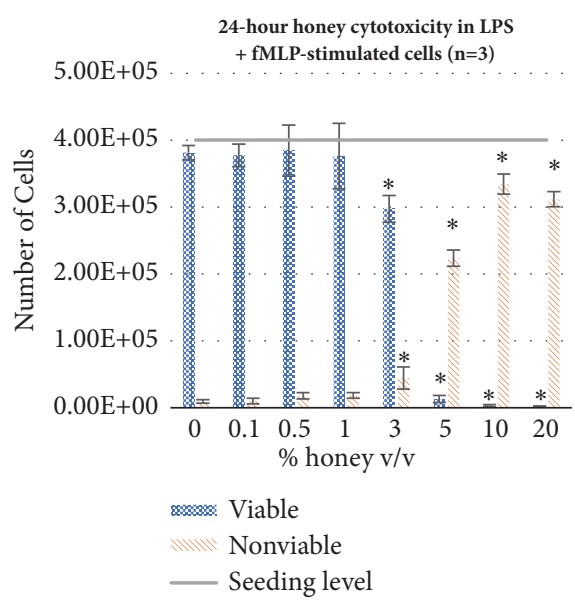

(d)

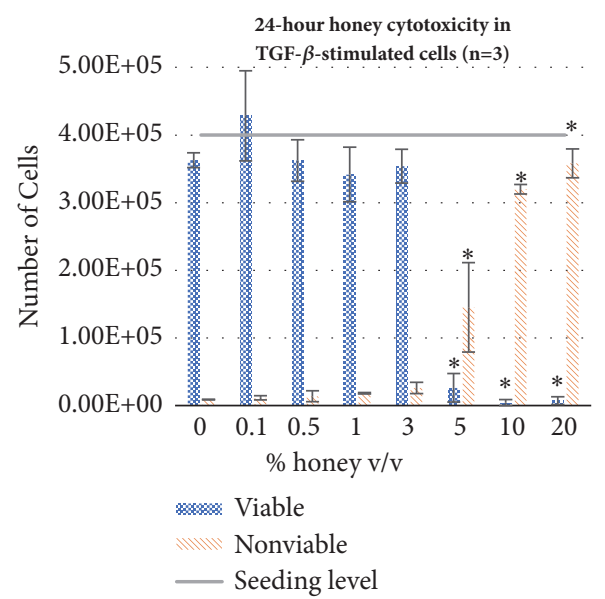

(f)

Figure 2: Viable and nonviable cell counts of NS, LPS + fMLP-stimulated, and TGF- $\beta$-stimulated dHL-60s cultured at each honey concentration for $3(\mathrm{a}, \mathrm{c}, \mathrm{e})$ and $24(\mathrm{~b}, \mathrm{~d}, \mathrm{f})$ hours. * indicates a statically significant difference from the respective $0 \%$ honey control of each cell type, viable or nonviable, at each timepoint. $\alpha=0.05$, measured via one-way ANOVA with Holm-Sidak post hoc.

3.5. I $\kappa \alpha \alpha$ Phosphorylation. Figure 5 displays the normalized $\mathrm{I} \kappa \mathrm{B} \alpha$ phosphorylation values for each sample type at 0 , 0.5 , and $3 \%$ honey. As expected, I $\kappa \mathrm{B} \alpha$ phosphorylation was significantly greater in the LPS + fMLP samples relative to the NS samples in the absence of honey. At $0.5 \%$ honey, $\mathrm{I} \kappa \mathrm{B} \alpha$ phosphorylation in the LPS + fMLP group was significantly lowered from the $0 \%$ honey samples, but still significantly greater than the NS samples. At $3 \%$ honey, $\mathrm{I} \kappa \mathrm{B} \alpha$ 


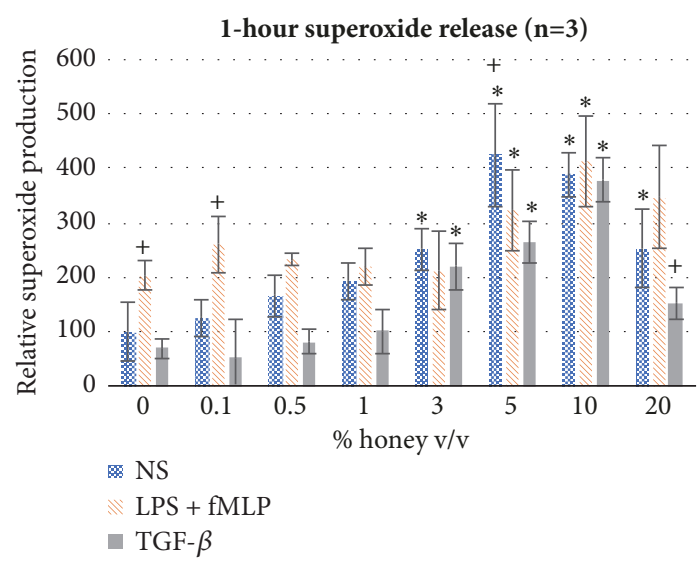

(a)

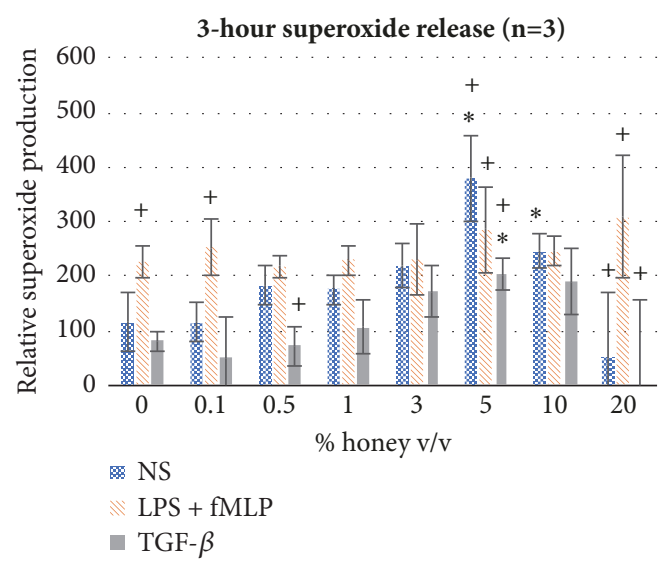

(b)

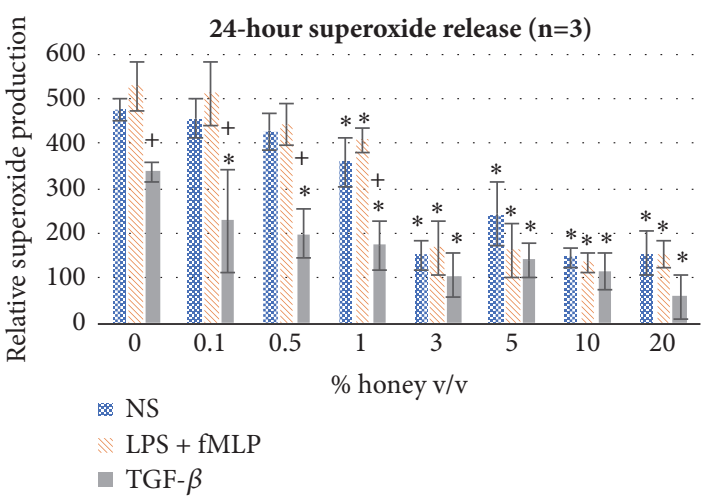

(c)

FIGURE 3: Superoxide production of the NS, LPS + fMLP-stimulated, and TGF- $\beta$-stimulated dHL-60s cultured at each honey concentration for (a) 1 , (b) 3, and (c) 24 hours. Values are shown relative to the $0 \%$ honey NS 1-hour value. * indicates statistical significance from the respective $0 \%$ honey value of that phenotype, and + indicates statistical significance from the other two phenotypes. $\alpha=0.05$, measured via two-way ANOVA with Holm-Sidak post hoc.

phosphorylation in the LPS + fMLP group was significantly lower than both the $0 \%$ and $0.5 \%$ samples and not significantly different from the NS group. These results indicate that Manuka honey lowers $\mathrm{I} \kappa \mathrm{B} \alpha$ phosphorylation in a dose-dependent fashion when activated via LPS and fMLP, reducing the activity of this inflammatory signal cascade. As expected, TGF- $\beta$ stimulation caused no significant difference in $\mathrm{I} \kappa \mathrm{B} \alpha$ phosphorylation relative to the NS group, and the presence of Manuka honey did not significantly affect $\mathrm{I} \kappa \mathrm{B} \alpha$ phosphorylation in the NS or TGF- $\beta$ group.

\section{Discussion}

The results of this study confirm the hypothesis that Manuka honey reduces neutrophil superoxide release, chemotaxis to $\mathrm{fMLP}$, and the activation of the NF- $\kappa \mathrm{B}$ pathway ( $\mathrm{I} \kappa \mathrm{B} \alpha$ phosphorylation) when present in concentrations under the cytotoxic limit. The MTS mitochondrial assay and the trypanexclusion assay establish a cytotoxic limit of Manuka honey beginning between 3-5\% v/v and increasing in cell death with increased honey concentration and time (Figures 1-2). Figure 3(a) indicates that concentrations of $3 \%$ honey and above cause an increase in superoxide release during the first hour of culture, suggesting that honey could amplify the initial acute inflammation response. However, Figure 3(c) demonstrates that, after 24 hours of culture, concentrations of $1 \%$ honey and above significantly reduce superoxide levels. From Figures 1-2, we know that concentrations of $5 \%$ honey and above cause cytotoxicity, which is likely the major contributor to the decrease in superoxide release at these honey levels. Figure 3(c) indicates a significant drop in superoxide release at the $1 \%$ honey level, which did not cause any cytotoxicity. Therefore, this drop at 1-3\% honey is likely due to honey reducing the superoxide output of the cells via anti-inflammatory effects, rather than cytotoxicity. The chemotaxis and $\mathrm{I} \kappa \mathrm{B} \alpha$ results also indicate that Manuka honey significantly reduces cell migration and $\mathrm{I} \kappa \mathrm{B} \alpha$ phosphorylation in a dose-dependent manner (Figures 4-5). Together, these experimental results indicate that Manuka honey has a significant anti-inflammatory effect on this in vitro neutrophil model.

These results agree with findings that have been previously published. Sell et al. conducted cytotoxicity testing using human dermal fibroblasts, human pulmonary microvascular endothelial cells, and human peripheral blood macrophages 


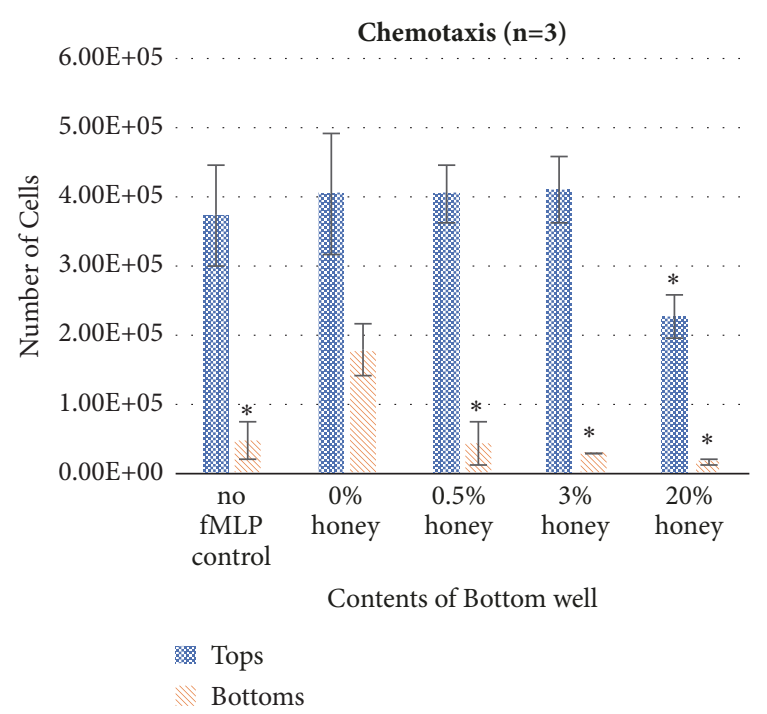

Figure 4: Chemotaxis of 500,000 dHL-60s to $50 \mathrm{nM}$ fMLP in various concentrations of honey. Cell numbers in top and bottom chambers were measured using a Countess II FL automated cell counter. * indicates a statistically significant difference in cell number from the $0 \%$ honey sample. $\alpha=0.05$, measured via one-way ANOVA with Holm-Sidak post hoc.

and observed a cytotoxic limit at 5\% v/v Manuka honey and above for all cell types, in line with our results [60]. Leong et al. tested the effect of several varieties of Manuka honeys on human neutrophil superoxide production and found that superoxide inhibition $\mathrm{IC}_{50}$ values range from 4.2 to $37.9 \mathrm{mg} / \mathrm{mL}$ when stimulated by $0.2 \mu \mathrm{g} / \mathrm{mL}$ phorbol 12 myristate 13-acetate (PMA). As neither the density of their Manuka honey varieties nor the time duration of the assay was reported, a direct comparison between their results and ours cannot be made. Knowing the density would allow the reported weight percent values to be converted to volume percent, enabling a direct comparison of their honey range to ours. However, both sets of results indicate a general trend of honey decreasing superoxide release. The Leong et al. study also involved the topical application of these honey samples to a murine arachidonic acid ear wound model, and they observed decreased neutrophil infiltration into the wound over a 4 -hour period after application [4]. This finding concurs with our chemotaxis assay results. A 2018 publication by Gasparrini et al. tested the effect of Manuka honey on NF$\kappa \mathrm{B}$ expression and $\mathrm{I} \kappa \mathrm{B} \alpha$ phosphorylation in LPS-stimulated RAW 264.7 macrophages and found that the honey reduced $\mathrm{I} \kappa \mathrm{B} \alpha$ phosphorylation and NF- $\kappa \mathrm{B}$ expression in these cells in a dose-dependent manner [61]. This study agrees with our $\mathrm{I} \kappa \mathrm{B} \alpha$ results, indicating that honey acts through the NF$\kappa \mathrm{B}$ pathway via $\mathrm{I} \kappa \mathrm{B} \alpha$ to reduce inflammatory behavior. Our results in this study thus broadly concur with the results of previously published literature.

The mechanisms through which Manuka honey affects the dHL-60 neutrophil model are unknown and possibly involve a combination of processes initiated by different
Manuka honey components. Alvarez-Suarez et al. have theorized that polyphenolic components of the honey, such as pinocembrin or pinobanksin, cross the cellular membrane to scavenge intracellular free radicals and trigger 5 ' AMP-activated protein kinase (AMPK) phosphorylation, increasing antioxidant enzyme expression [10]. Evidence gathered by Gasparrini et al. demonstrates that Manuka honey increases the intracellular expression of antioxidant enzymes such as glutathione peroxidase, glutathione reductase, and glutathione s-transferase in macrophages, supporting the Alvarez-Suarez theory [61]. Ultimately, more work is necessary to fully elucidate the mechanisms of action of Manuka honey on neutrophils and other relevant cell types.

Despite the fact that these studies were conducted in vitro, certain inferences can be made regarding the role Manuka honey plays in the wound site. When Manuka honey is used clinically as a wound treatment, it is typically either daubed directly onto the wound and covered with a bandage, soaked into a cloth dressing which is then covered with a secondary dry dressing to fasten the honey dressing to the wound, or incorporated into a hydrogel dressing [62]. These methods of application cause the surface of the wound to experience a high concentration of Manuka honey, potentially in excess of the $20 \% \mathrm{v} / \mathrm{v}$ honey concentration used as the highest end of the honey concentration range in this study. Given the cytotoxicity results reported in this study, it is likely that the surface of the wound experiences a "zone of death," where the bactericidal and osmotic effects combine to kill not only foreign bacteria, but also native human cells, including large numbers of neutrophils which arrive in the wound soon after injury [60]. As the honey diffuses down into the wound environment and becomes more dilute, it is possible that the deeper wound environment encounters honey concentrations closer to the $0.5 \%$ and $3 \%$ concentrations investigated in this study. The exact concentrations of the honey at relevant penetration depths within the wound are speculative and have yet to be measured in vivo. However, according to this study, such honey concentrations would initially promote the neutrophils in the wound to release superoxide, amplifying the acute response within the first hour of application. However, as time continues, this trend reverses, causing the neutrophils to attenuate their superoxide release in the presence of the honey. Meanwhile, the honey's osmotic potential starts a slow net flow of exudate from the deep tissue through the wound bed and out to the wound surface, washing debris and bacteria from the wound site. It is possible that this flow also carries the neutrophils in the wound up to the cytotoxic "zone of death," and as they are killed, they are initially replaced by naïve neutrophils through the bloodstream. However, as shown by the chemotaxis results in this study, the presence of honey decreases neutrophil chemotaxis in response to the fMLP. Additionally, the I $\kappa \mathrm{B} \alpha$ phosphorylation results indicate that the honey will begin downregulating the NF- $\kappa \mathrm{B}$ pathway, reducing the expression of inflammatory behaviors in neutrophils in the presence of inflammatory stimuli like LPS and fMLP. Thus, in a honey-treated wound, the time of the acute inflammation phase is likely shortened, with 


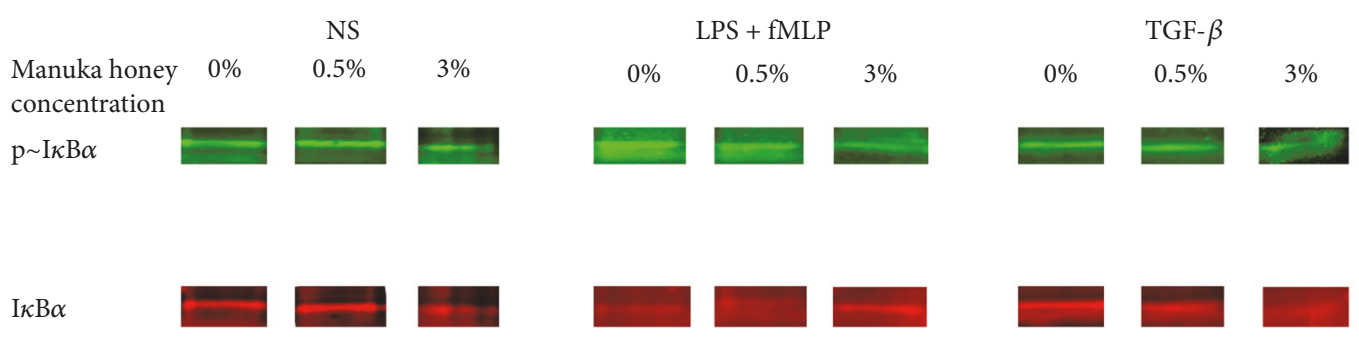

(a)

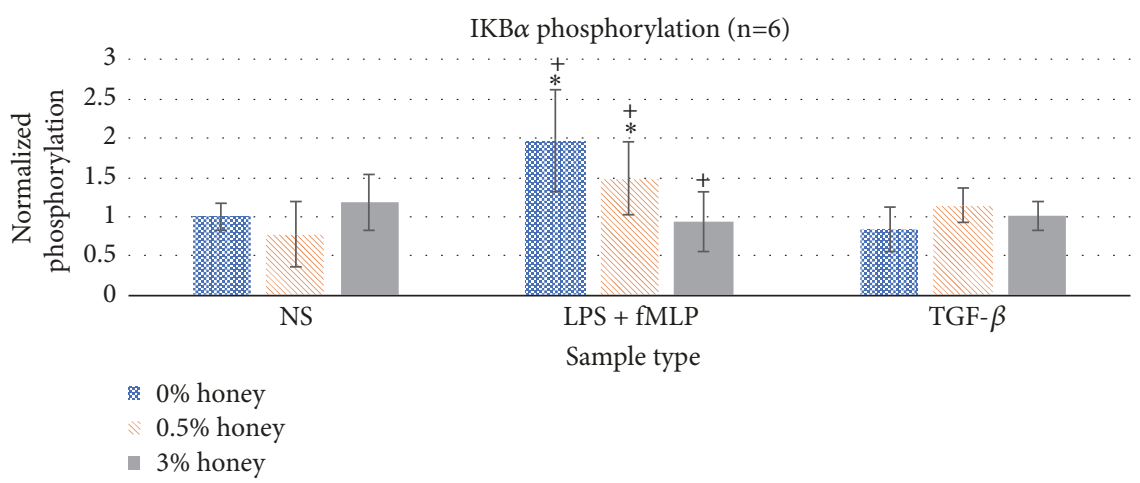

(b)

Figure 5: (a) Representative Western blot bands of $\mathrm{p} \sim \mathrm{I} \kappa \mathrm{B} \alpha$ and total $\mathrm{I} \kappa \mathrm{B} \alpha$ for each sample type. (b) I $\kappa \mathrm{B} \alpha$ phosphorylation expressed as the ratio of $\mathrm{p} \sim \mathrm{I} \kappa \mathrm{B} \alpha$ to total $\mathrm{I} \kappa \mathrm{B} \alpha$, normalized to NS $0 \%$ honey control. * indicates a statistically significant difference from the NS control at that respective honey level. + indicates a statistically significant difference from the other concentrations of honey for that stimulus group. $\alpha=0.05$, measured via two-way ANOVA with Holm-Sidak post hoc.

fewer and fewer neutrophils chemotaxing to the wound site over time and a reduced inflammation response in the cells encountering inflammatory stimuli.

In addition to their role in wound inflammation, neutrophils also play a part in several inflammation-related pathologies $[26,63]$. Excessive neutrophil activity contributes to the formation of atherosclerotic plaque [63], tissue damage associated with chronic obstructive pulmonary disorder [64], tumor formation [65], and inflammatory bowel disease [66-68], among other conditions. The ability of Manuka honey to decrease neutrophil recruitment and inflammatory behavior represents a potential therapeutic opportunity for these pathologies. In particular, a 2008 study by Prakash et al. demonstrated that oral administration of Manuka honey significantly reduced colonic inflammation in a rat inflammatory bowel disease model [69]. Although this specific application has yet to be replicated in humans, this study demonstrates the promise of Manuka honeybased therapies in treating inflammation-related pathologies.

The results detailed in this paper demonstrate in vitro the specific effects of Manuka honey that enable it to shorten and resolve wound inflammation in vivo. They also point towards possible advantages of a lower level, longer term controlled release delivery of the honey to avoid counterproductive cytotoxic effects. As these results show, the inflammation-resolving effects of Manuka honey are present at and below the 3\% v/v level. As such, a controlled release of $3 \% \mathrm{v} / \mathrm{v}$ or below Manuka honey from an implanted tissue engineering template would minimize inflammation around the template, allowing faster and more complete healing and tissue-template integration. This effect makes Manuka honey a useful addition to templates for a wide variety of tissue regeneration applications.

Future research will involve examining the effect of Manuka honey on the release of molecular signals (proinflammatory, anti-inflammatory, chemokines, matrix-degrading, and proangiogenic) from this neutrophil model. Additionally, Manuka honey will be incorporated into electrospun tissue templates to examine the effect of honey incorporation on neutrophil NETosis. These experiments will create a better understanding of how honey affects the orchestration of the inflammatory, angiogenic, and inflammation-resolving processes within the wound. As the degree of NETosis has been demonstrated to correlate with fibrous capsule formation and rejection of implanted templates in vivo, the NETosis experiments will also inform our understanding of the potential of Manuka honey to improve tissue-template integration and reduce capsule formation. Future research should also include investigation into the effects of other honey varieties on neutrophil inflammatory behaviors. While Manuka honey is the current focus of this paper due to its prevalence in the wound healing field, it is possible that other honey varieties may be as or even more effective at reducing neutrophil inflammatory behaviors. An in vivo wound healing model should also be used to confirm the effects described in this paper and measure the honey concentration gradient within the wound. 


\section{Conclusions}

In this study, the effect of various concentrations of Manuka honey on NS, LPS + fMLP, and TGF- $\beta$-stimulated dHL60 neutrophil models was observed in several ways. First, a moderate cytotoxic effect was found to begin at 3-5\% honey and become stronger as honey concentration increased. Concentrations of Manuka honey at 3\% and above were found to amplify superoxide production in the first 1-3 hours of culture, but then they suppress superoxide by 24 hours of culture. Furthermore, concentrations of $0.5 \%$ and above were found to significantly suppress chemotaxis to fMLP and reduce $\mathrm{I} \kappa \mathrm{B} \alpha$ phosphorylation. These results suggest that Manuka honey has an anti-inflammatory effect on neutrophils, reducing their recruitment to the wound site, their superoxide production, and their intracellular inflammatory signalling.

\section{Data Availability}

The data used to support the findings of this study are included within the supplementary information files.

\section{Conflicts of Interest}

The authors declare that there are no conflicts of interest regarding this paper.

\section{Acknowledgments}

The research reported in this publication was supported by the National Institute of Biomedical Imaging and Bioengineering of the National Institutes of Health under Award Number R15EB022345. This research was also funded by the Van Vleet Memorial Doctoral Award of the University of Memphis. The authors thank the University of Memphis and the University of Tennessee Health Science Campus, as well as Dr. Judith Cole for assistance with Western blot troubleshooting.

\section{Supplementary Materials}

Supplementary materials are available on the journal website detailing the differentiation of the HL-60 cells and preliminary Western blots used to establish a timeframe for $\mathrm{I} \kappa \mathrm{B} \alpha$ phosphorylation. Additionally, Excel files are provided with the raw data obtained from each experiment described in this paper. (Supplementary Materials)

\section{References}

[1] G. T. Gethin, S. Cowman, and R. M. Conroy, "The impact of Manuka honey dressings on the surface $\mathrm{pH}$ of chronic wounds," International Wound Journal, vol. 5, no. 2, pp. 185-194, 2008.

[2] R. A. Cooper, P. C. Molan, and K. G. Harding, "The sensitivity to honey of Gram-positive cocci of clinical significance isolated from wounds," Journal of Applied Microbiology, vol. 93, no. 5, pp. 857-863, 2002.
[3] M. S. Osato, S. G. Reddy, and D. Y. Graham, "Osmotic effect of honey on growth and viability of Helicobacterpylori," Digestive Diseases and Sciences, vol. 44, no. 3, pp. 462-464, 1999.

[4] A. G. Leong, P. M. Herst, and J. L. Harper, "Indigenous New Zealand honeys exhibit multiple anti-inflammatory activities," Journal of Innate Immunity, vol. 18, no. 3, pp. 459-466, 2012.

[5] P. C. Molan, "Re-introducing honey in the management of wounds and ulcers-theory and practice," Ostomy Wound Management, vol. 48, no. 11, pp. 28-40, 2002.

[6] J. Chirife, G. Scarmato, and L. Herszage, "Scientific basis for use of granulated sugar in treatment of infected wounds," The Lancet, vol. 319, no. 8271, pp. 560-561, 1982.

[7] M. Kassim, M. Achoui, M. R. Mustafa, M. A. Mohd, and K. M. Yusoff, "Ellagic acid, phenolic acids, and flavonoids in Malaysian honey extracts demonstrate in vitro anti-inflammatory activity," Nutrition Research, vol. 30, no. 9, pp. 650-659, 2010.

[8] A. J. van den Berg, E. van den Worm, H. C. Q. van Ufford, S. B. Halkes, M. J. Hoekstra, and C. J. Beukelman, "An in vitro examination of the antioxidant and anti-inflammatory properties of buckwheat honey," Journal of Wound Care, vol. 17, no. 4, pp. 172 178, 2008.

[9] M. Candiracci, E. Piatti, M. Dominguez-Barragán et al., "Anti-inflammatory activity of a honey flavonoid extract on lipopolysaccharide-activated N13 microglial cells," Journal of Agricultural and Food Chemistry, vol. 60, no. 50, pp. 1230412311, 2012.

[10] J. M. Alvarez-Suarez, F. Giampieri, M. Cordero et al., "Activation of AMPK/Nrf2 signalling by Manuka honey protects human dermal fibroblasts against oxidative damage by improving antioxidant response and mitochondrial function promoting wound healing," Journal of Functional Foods, vol. 25, pp. 38-49, 2016.

[11] K. Brudzynski, K. Abubaker, L. St-Martin, and A. Castle, "Reexamining the role of hydrogen peroxide in bacteriostatic and bactericidal activities of honey," Frontiers in Microbiology, vol. 2, article 213, 2011.

[12] L. M. Bang, C. Buntting, and P. Molan, "The effect of dilution on the rate of hydrogen peroxide production in honey and its implications for wound healing," The Journal of Alternative and Complementary Medicine, vol. 9, no. 2, pp. 267-273, 2003.

[13] J. W. White Jr., M. H. Subers, and A. I. Schepartz, “The identification of inhibine, the antibacterial factor in honey, as hydrogen peroxide and its origin in a honey glucose-oxidase system," Biochimica et Biophysica Acta, vol. 73, no. 1, pp. 57-70, 1963.

[14] B. Minden-Birkenmaier and G. Bowlin, "Honey-based templates in wound healing and tissue engineering," Bioengineering, vol. 5 , no. 2, p. 46, 2018.

[15] P. C. Molan, R. Cooper, and R. White, "Why honey works," in Honey in Modern Wound Management, vol. 9, pp. 36-37, Wounds UK Ltd, Aberdeen, Scotland, 2009.

[16] P. C. Molan, "The antibacterial activity of honey: 2. Variation in the potency of the antibacterial activity," Bee World, vol. 73, no. 2, pp. 59-76, 1992.

[17] P. C. Molan, "Potential of honey in the treatment of wounds and burns," American Journal of Clinical Dermatology, vol. 2, no. 1, pp. 13-19, 2001.

[18] P. C. Molan, "The evidence supporting the use of honey as a wound dressing," The International Journal of Lower Extremity Wounds, vol. 5, no. 1, pp. 40-54, 2006. 
[19] A. Tonks, R. A. Cooper, A. J. Price, P. C. Molan, and K. P. Jones, "Stimulation of TNF- $\alpha$ release in monocytes by honey," Cytokine, vol. 14, no. 4, pp. 240-242, 2001.

[20] A. Oryan, E. Alemzadeh, and A. Moshiri, "Biological properties and therapeutic activities of honey in wound healing: a narrative review and meta-analysis," Journal of Tissue Viability, vol. 25, no. 2, pp. 98-118, 2016.

[21] B. Minden-Birkenmaier, "Preliminary investigation and characterization of electrospun polycaprolactone and Manuka honey scaffolds for dermal repair," Journal of Engineered Fabrics \& Fibers, vol. 10, no. 4, 2015.

[22] K. R. Hixon, T. Lu, M. N. Carletta, S. H. McBride-Gagyi, B. E. Janowiak, and S. A. Sell, "A preliminary in vitro evaluation of the bioactive potential of cryogel scaffolds incorporated with Manuka honey for the treatment of chronic bone infections," Journal of Biomedical Materials Research Part B: Applied Biomaterials, vol. 106, no. 5, pp. 1918-1933, 2018.

[23] K. R. Hixon, T. Lu, S. H. McBride-Gagyi, B. E. Janowiak, and S. A. Sell, "A comparison of tissue engineering scaffolds incorporated with Manuka honey of varying UMF," BioMed Research International, vol. 2017, Article ID 4843065, 12 pages, 2017.

[24] P. U. Kadakia, E. A. Growney Kalaf, A. J. Dunn, L. P. Shornick, and S. A. Sell, "Comparison of silk fibroin electrospun scaffolds with poloxamer and honey additives for burn wound applications," Journal of Bioactive and Compatible Polymers, vol. 33, no. 1, pp. 79-94, 2018.

[25] X. Yang, L. Fan, L. Ma et al., "Green electrospun Manuka honey/ silk fibroin fibrous matrices as potential wound dressing," Materials and Corrosion, vol. 119, pp. 76-84, 2017.

[26] G. S. Selders, A. E. Fetz, M. Z. Radic, and G. L. Bowlin, "An overview of the role of neutrophils in innate immunity, inflammation and host-biomaterial integration," Regenerative Biomaterials, vol. 4, no. 1, pp. 55-68, 2017.

[27] R. Jenkins, N. Burton, and R. Cooper, "Effect of Manuka honey on the expression of universal stress protein A in meticillin-resistant Staphylococcus aureus," International Journal of Antimicrobial Agents, vol. 37, no. 4, pp. 373-376, 2011.

[28] A. Wallace, S. Eady, M. Miles et al., "Demonstrating the safety of Manuka honey $\mathrm{UMF}^{\circledR} 20+$ in a human clinical trial with healthy individuals," British Journal of Nutrition, vol. 103, no. 7, pp. 10231028, 2010.

[29] T. S. Blackwell, T. R. Blackwell, E. P. Holden, B. W. Christman, and J. W. Christman, "In vivo antioxidant treatment suppresses nuclear factor-kappa B activation and neutrophilic lung inflammation," The Journal of Immunology, vol. 157, no. 4, pp. 16301637, 1996.

[30] P. A. Baeuerle and V. R. Baichwal, "NF- $\kappa$ B as a frequent target for immunosuppressive and anti-inflammatory molecules," Advances in Immunology, vol. 65, pp. 111-137, 1997.

[31] J. V. Dovi, A. M. Szpaderska, and L. A. DiPietro, "Neutrophil function in the healing wound: Adding insult to injury?" Thrombosis and Haemostasis-Stuttgart, vol. 92, no. 2, pp. 275280, 2004.

[32] B. W. Bardoel, E. F. Kenny, G. Sollberger, and A. Zychlinsky, "The balancing act of neutrophils," Cell Host \& Microbe, vol. 15, no. 5, pp. 526-536, 2014.

[33] S. De Oliveira, E. E. Rosowski, and A. Huttenlocher, "Neutrophil migration in infection and wound repair: going forward in reverse," Nature Reviews Immunology, vol. 16, no. 6, pp. 378391, 2016.
[34] J. V. Dovi, L.-K. He, and L. A. DiPietro, "Accelerated wound closure in neutrophil-depleted mice," Journal of Leukocyte Biology, vol. 73, no. 4, pp. 448-455, 2003.

[35] H. R. Jones, C. T. Robb, M. Perretti, and A. G. Rossi, “The role of neutrophils in inflammation resolution," Seminars in Immunology, vol. 28, no. 2, pp. 137-145, 2016.

[36] M. I. Cuartero, I. Ballesteros, A. Moraga et al., "N2 neutrophils, novel players in brain inflammation after stroke: modulation by the ppar $\gamma$ agonist rosiglitazone," Stroke, vol. 44, no. 12, pp. 34983508, 2013.

[37] Z. G. Fridlender, J. Sun, S. Kim et al., "Polarization of tumorassociated neutrophil phenotype by TGF- $\beta$ : "N1" versus "N2" TAN," Cancer Cell, vol. 16, no. 3, pp. 183-194, 2009.

[38] V. C. Ardi, T. A. Kupriyanova, E. I. Deryugina, and J. P. Quigley, "Human neutrophils uniquely release TIMP-free MMP-9 to provide a potent catalytic stimulator of angiogenesis," Proceedings of the National Acadamy of Sciences of the United States of America, vol. 104, no. 51, pp. 20262-20267, 2007.

[39] S. Patel and S. Cichello, "Manuka honey: an emerging natural food with medicinal use," Natural Products and Bioprospecting, vol. 3, no. 4, pp. 121-128, 2013.

[40] Q. J. Wang, "PKD at the crossroads of DAG and PKC signaling," Trends in Pharmacological Sciences, vol. 27, no. 6, pp. 317-323, 2006.

[41] H. Gao, Y. Sun, Y. Wu et al., "Identification of $\beta$-arrestin2 as a $G$ protein-coupled receptor-stimulated regulator of NF- $\kappa \mathrm{B}$ pathways," Molecular Cell, vol. 14, no. 3, pp. 303-317, 2004.

[42] M. J. Rane and J. B. Klein, "Regulation of neutrophil apoptosis by modulation of $\mathrm{PKB} / \mathrm{Akt}$ activation," Frontiers in Bioscience, vol. 14, no. 7, pp. 2400-2412, 2009.

[43] F. A. Grieco, F. Vendrame, I. Spagnuolo, and F. Dotta, "Innate immunity and the pathogenesis of type 1 diabetes," Seminars in Immunopathology, vol. 33, no. 1, pp. 57-66, 2011.

[44] P. Viatour, M. Merville, V. Bours, and A. Chariot, "Phosphorylation of NF- $\kappa \mathrm{B}$ and $\mathrm{I} \kappa \mathrm{B}$ proteins: implications in cancer and inflammation," Trends in Biochemical Sciences, vol. 30, no. 1, pp. 43-52, 2005.

[45] T. Lawrence, "The nuclear factor NF- $\kappa$ B pathway in inflammation," Cold Spring Harbor Perspectives in Biology, vol. 1, no. 6, Article ID a001651, 2009.

[46] M. S. Hayden and S. Ghosh, "Shared principles in NF- $\kappa$ B signaling," Cell, vol. 132, no. 3, pp. 344-362, 2008.

[47] R. Gallagher, S. Collins, J. Trujillo et al., "Characterization of the continuous, differentiating myeloid cell line (HL-60) from a patient with acute promyelocytic leukemia," Blood, vol. 54, no. 3, pp. 713-733, 1979.

[48] S. J. Collins, F. W. Ruscetti, R. E. Gallagher, and R. C. Gallo, "Normal functional characteristics of cultured human promyelocytic leukemia cells (HL-60) after induction of differentiation by dimethylsulfoxide," The Journal of Experimental Medicine, vol. 149, no. 4, pp. 969-974, 1979.

[49] S. J. Collins, "The HL-60 promyelocytic leukemia cell line: Proliferation, differentiation, and cellular oncogene expression," Blood, vol. 70, no. 5, pp. 1233-1244, 1987.

[50] B. H. Ewert, J. C. Jennette, and R. J. Falk, "Anti-myeloperoxidase antibodies stimulate neutrophils to damage human endothelial cells," Kidney International, vol. 41, no. 2, pp. 375-383, 1992.

[51] S. Trellakis, H. Farjah, K. Bruderek et al., "Peripheral blood neutrophil granulocytes from patients with head and neck squamous cell carcinoma functionally differ from their counterparts in healthy donors," International Journal of Immunopathology and Pharmacology, vol. 24, no. 3, pp. 683-693, 2011. 
[52] M. G. Tonneson, L. A. Smedly, and P. M. Henson, "Neutrophilendothelial cell interactions. Modulation of neutrophil adhesiveness induced by complement fragments C5a and C5a des arg and formyl-methionyl-leucyl-phenylalanine in vitro," The Journal of Clinical Investigation, vol. 74, no. 5, pp. 1581-1592, 1984.

[53] S. J. Martin, J. G. Bradley, and T. G. Cotter, "HL-60 cells induced to differentiate towards neutrophils subsequently die via apoptosis," Clinical \& Experimental Immunology, vol. 79, no. 3, pp. 448-453, 1990.

[54] J. Nath and A. Powledge, "Modulation of human neutrophil inflammatory responses by nitric oxide: Studies in unprimed and LPS-primed cells," Journal of Leukocyte Biology, vol. 62, no. 6, pp. 805-816, 1997.

[55] R. A. Fava, N. J. Olsen, A. E. Postlethwaite et al., "Transforming growth factor $\beta 1$ (TGF- $\beta 1$ ) induced neutrophil recruitment to synovial tissues: Implications for TGF- $\beta$-driven synovial inflammation and hyperplasia," The Journal of Experimental Medicine, vol. 173, no. 5, pp. 1121-1132, 1991.

[56] D. T. Cromack, M. B. Sporn, A. B. Roberts, M. J. Merino, L. L. Dart, and J. A. Norton, "Transforming growth factor $\beta$ levels in rat wound chambers," Journal of Surgical Research, vol. 42, no. 6, pp. 622-628, 1987.

[57] Y. Chen and W. G. Junger, "Measurement of oxidative burst in neutrophils," Methods in Molecular Biology, vol. 844, pp. 115124, 2012.

[58] H. J. Cohen and M. E. Chovaniec, "Superoxide generation by digitonin-stimulated guinea pig granulocytes," The Journal of Clinical Investigation, vol. 61, no. 4, pp. 1081-1087, 1978.

[59] N. Lehman, M. Di Fulvio, N. McCray, I. Campos, F. Tabatabaian, and J. Gomez-Cambronero, "Phagocyte cell migration is mediated by phospholipases PLD1 and PLD2," Blood, vol. 108, no. 10, pp. 3564-3572, 2006.

[60] S. A. Sell, P. S. Wolfe, A. J. Spence et al., "A preliminary study on the potential of Manuka honey and platelet-rich plasma in wound healing," International Journal of Biomaterials, vol. 2012, Article ID 313781, 14 pages, 2012.

[61] M. Gasparrini, S. Afrin, T. Y. Forbes-Hernández et al., "Protective effects of Manuka honey on LPS-treated RAW 264.7 macrophages. Part 2: Control of oxidative stress induced damage, increase of antioxidant enzyme activities and attenuation of inflammation," Food and Chemical Toxicology, vol. 120, pp. 578-587, 2018.

[62] P. C. Molan and J. A. Betts, "Clinical usage of honey as a wound dressing: an update," Journal of Wound Care, vol. 13, no. 9, pp. 353-356, 2004

[63] M. G. Ionita, P. Van Den Borne, L. M. Catanzariti et al., "High neutrophil numbers in human carotid atherosclerotic plaques are associated with characteristics of rupture-prone lesions," Arteriosclerosis, Thrombosis, and Vascular Biology, vol. 30, no. 9, pp. 1842-1848, 2010.

[64] S. D. Lucas, E. Costa, R. C. Guedes, and R. Moreira, “Targeting COPD: Advances on low-molecular-weight inhibitors of human neutrophil elastase," Medicinal Research Reviews, vol. 33, no. 1, pp. E73-E101, 2013.

[65] A. M. Houghton, D. M. Rzymkiewicz, H. Ji et al., "Neutrophil elastasemediated degradation of IRS-1 accelerates lung tumor growth," Nature Medicine, vol. 16, no. 2, p. 219, 2010.

[66] T. Kucharzik, S. V. Walsh, J. Chen, C. A. Parkos, and A. Nusrat, "Neutrophil transmigration in inflammatory bowel disease is associated with differential expression of epithelial intercellular junction proteins," The American Journal of Pathology, vol. 159, no. 6, pp. 2001-2009, 2001.

[67] P. Sharon and W. F. Stenson, "Enhanced synthesis of leukotriene B4 by colonic mucosa in inflammatory bowel disease," Gastroenterology, vol. 86, no. 3, pp. 453-460, 1984.

[68] A. E. Brannigan, P. R. O’Connell, H. Hurley et al., "Neutrophil apoptosis is delayed in patients with inflammatory bowel disease," Shock, vol. 13, no. 5, pp. 361-366, 2000.

[69] A. Prakash, B. Medhi, P. K. Avti, U. N. Saikia, P. Pandhi, and K. L. Khanduja, "Effect of different doses of Manuka honey in experimentally induced inflammatory bowel disease in rats," Phytotherapy Research, vol. 22, no. 11, pp. 1511-1519, 2008. 


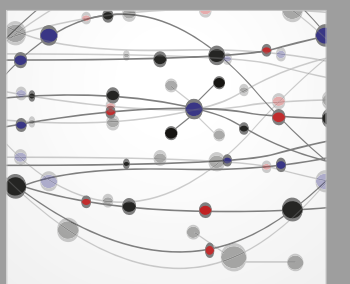

The Scientific World Journal
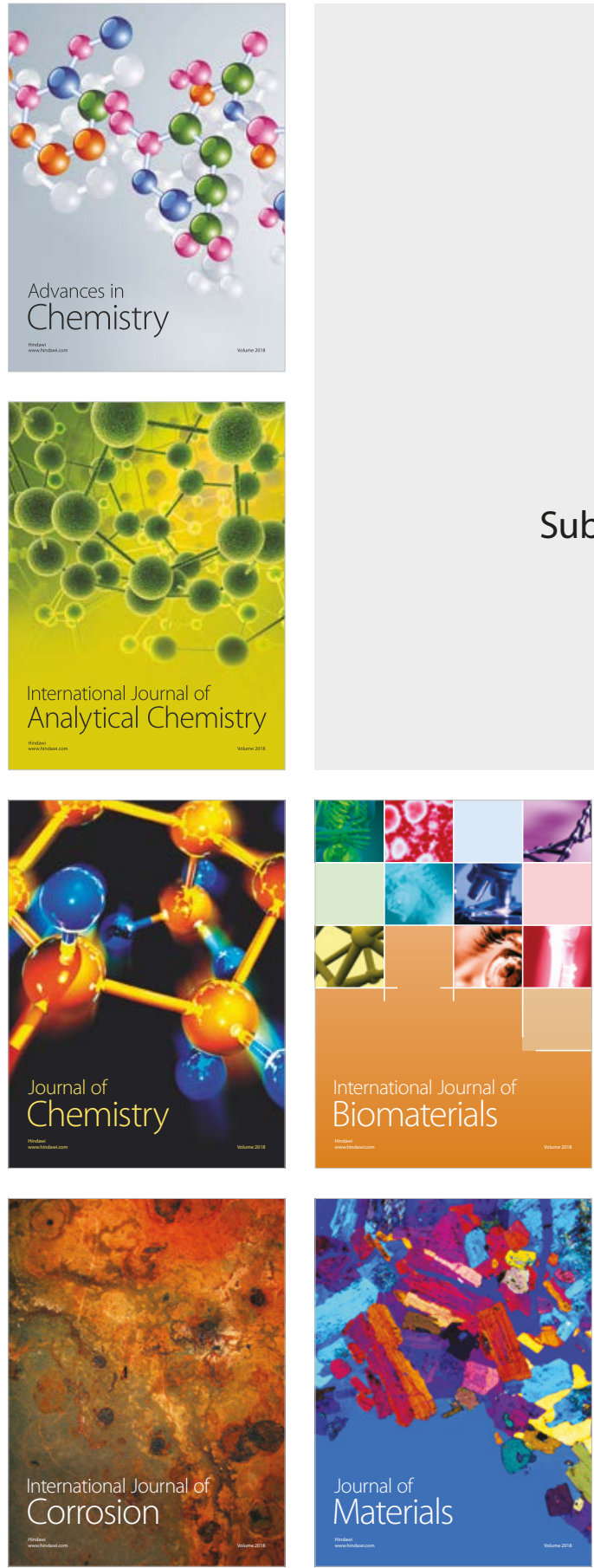

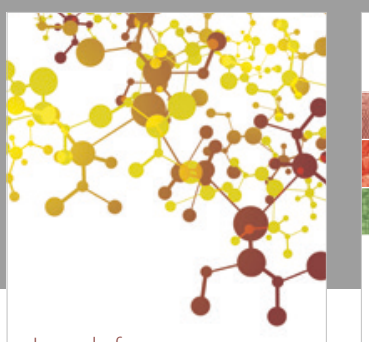

Journal of

Applied Chemistry
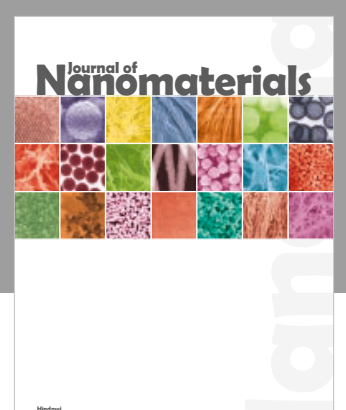

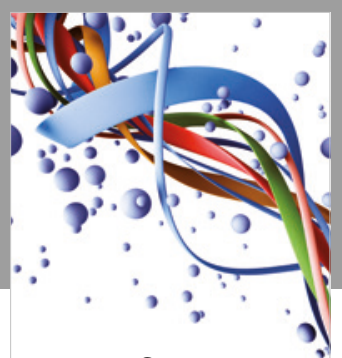

Scientifica

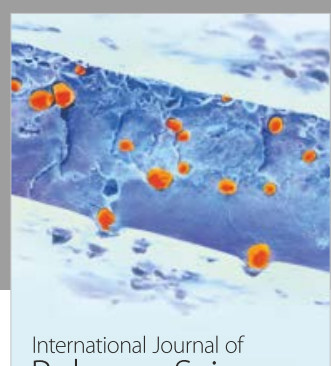

Polymer Science

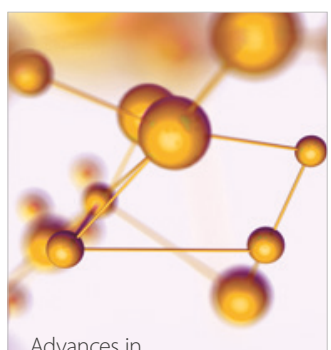

Physical Chemistry
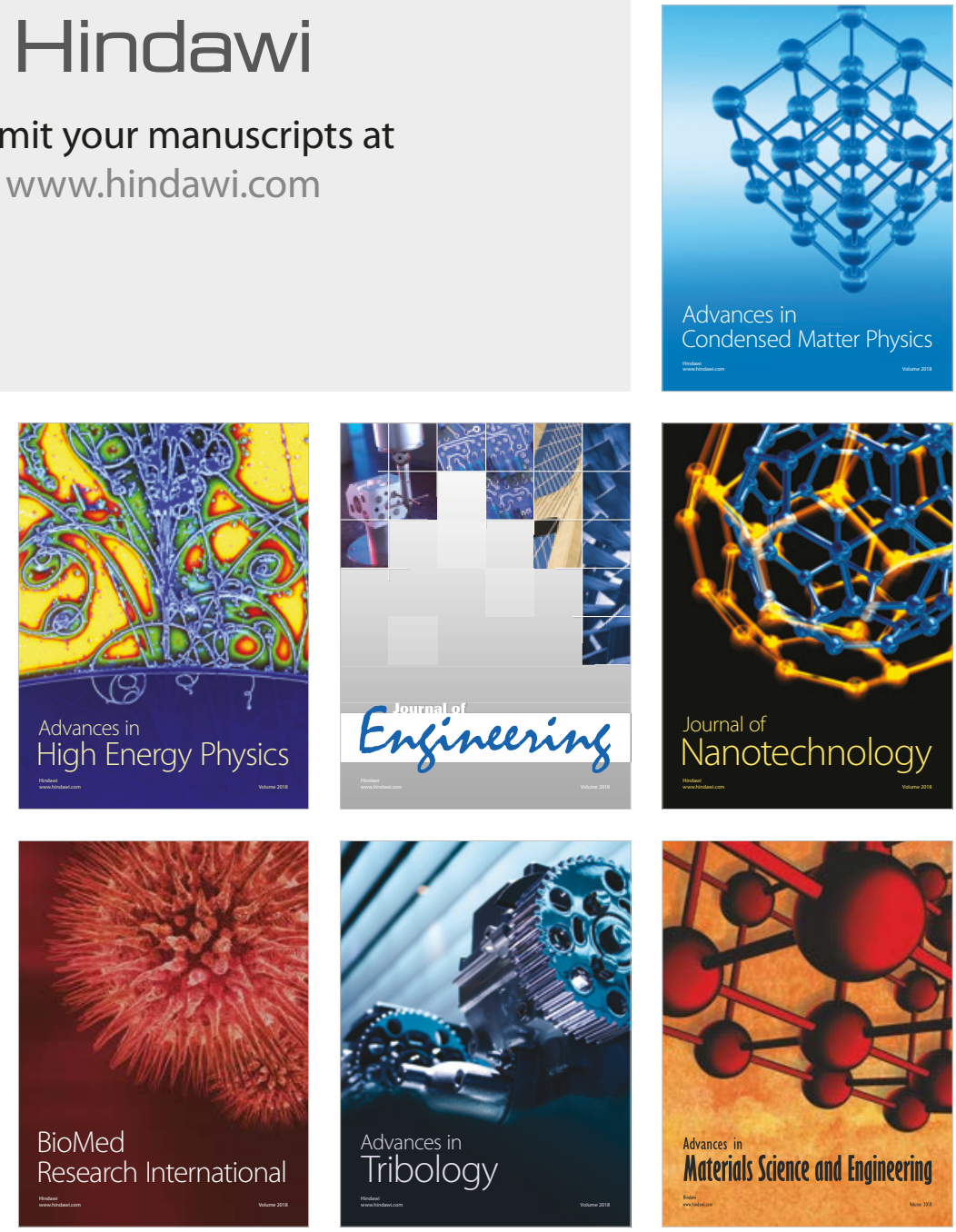